\title{
Telmisartan, a unique ARB, improves left ventricular remodeling of infarcted heart by activating PPAR gamma
}

\author{
Yasuhiro Maejima ${ }^{1}$, Hiroyuki Okada ${ }^{1}$, Go Haraguchi ${ }^{1}$, Yasuyuki Onai ${ }^{1}$, Hisanori Kosuge ${ }^{1}$, Jun-ichi Suzuki ${ }^{2}$ \\ and Mitsuaki Isobe ${ }^{1}$
}

Unfavorable left ventricular (LV) remodeling after myocardial infarction (MI) leads to cardiac dysfunction. We examined whether Telmisartan, an angiotensin (Ang) II type I receptor blocker (ARB), could improve the recovery of LV function in a rat model of MI. The effect of Telmisartan as a peroxisome proliferator-activated receptor- $\gamma$ (PPAR- $\gamma$ ) agonist was also investigated. After 28 days of $\mathrm{Ml}$, a significant improvement of survival was observed in the Telmisartan-treated rat group compared with the vehicle control rat group, non-PPAR- $\gamma$ agonistic ARB (Losartan)-treated rat group, and Telmisartan plus specific PPAR- $\gamma$ antagonist (GW9662)-treated rat group. Although no significant differences of blood pressure or infarct size were observed among these four groups, the Telmisartan group had better systolic and diastolic LV function. There was a significant reduction of the plasma brain natriuretic peptide level, cardiac fibrosis area, infiltration of macrophages, size of cardiomyocytes, terminal deoxynucleotidyl transferase dUTP nick end labeling-positive myocytes, activation of matrix metalloproteinases-2 and -9 (MMPs-2/9), and expression of transforming growth factor $\beta-1$ (TGF- $\beta 1$ ), connective tissue growth factor (CTGF), and osteopontin (OPN), while expression of PPAR- $\gamma$ and activation of tissue inhibitor of metalloproteinase-1 (TIMP-1) was enhanced, in the noninfarcted myocardium of rats from the Telmisartan group compared with the other three groups. To mimic ischemic conditions in vitro, neonatal rat cardiomyocytes and cardiac fibroblasts were incubated in hypoxic condition for $24 \mathrm{~h}$. Increased transcriptional activation of PPAR- $\gamma$ and TIMP-1, and inhibition of TGF- $\beta 1$ expression were observed in cardiomyocytes, while decreased activation of MMPs- $2 / 9$ and decrease in CTGF and OPN expression was seen in cardiac fibroblasts cultured with Telmisartan. In conclusion, Telmisartan prevented unfavorable cardiac remodeling through a reduction of cardiac hypertrophy and fibrosis. An anti-inflammatory effect and PPAR- $\gamma$ activation were suggested to be important in addition to suppression of Ang II activity.

Laboratory Investigation (2011) 91, 932-944; doi:10.1038/labinvest.2011.45; published online 14 March 2011

KEYWORDS: angiotensin II; LV remodeling; MMP; PPAR- $\gamma ;$ TGF- $\beta 1$

Unfavorable left ventricular (LV) remodeling after myocardial infarction (MI) is characterized by hypertrophy associated with interstitial fibrosis of the noninfarcted myocardium, and causes LV dilatation and cardiac dysfunction. It is known that angiotensin II (Ang II) has a crucial role in the development of unfavorable LV remodeling, and recent studies based on animal experiments have demonstrated that treatment with an Ang-converting enzyme inhibitor (ACE-I) and/or Ang II type I receptor blocker (ARB) can attenuate unfavorable LV remodeling. ${ }^{1,2}$ Moreover, other experimental studies suggested that activation of peroxisome proliferator-activated receptor- $\gamma$ (PPAR- $\gamma)$, a transcription factor, improves unfavorable LV remodeling after MI. ${ }^{3-5}$

PPAR- $\gamma$ agonists not only regulate insulin sensitivity, but also have an anti-inflammatory effect by inhibiting the expression of adhesion molecules, cytokines, and chemokines, as well as anti-fibrotic activity by inhibiting transforming growth factor $\beta$-1 (TGF- $\beta 1$ ) signaling, and the activity of matrix metalloprotease (MMP)-2/9 and the osteopontin (OPN) ${ }^{6-9}$ Telmisartan is a structurally unique ARB that acts as a partial PPAR- $\gamma$ agonist (activating the receptor $25-30 \%$

\footnotetext{
'Department of Cardiovascular Medicine, Tokyo Medical and Dental University, 1-5-45 Yushima, Tokyo, Japan and ${ }^{2}$ Department of Advanced Clinical Science and Therapeutics, University of Tokyo, 7-3-1 Hongo, Tokyo, Japan

Correspondence: Professor M Isobe, MD, PhD, Department of Cardiovascular Medicine, Tokyo Medical and Dental University, 1-5-45 Yushima, Bunkyo, Tokyo 113-8519, Japan.

E-mail: isobemi.cvm@tmd.ac.jp

Received 22 June 2010; revised 29 November 2010; accepted 3 January 2011
} 
of the maximum level achieved by full PPAR- $\gamma$ agonists) as well as an $A R B,{ }^{10}$ thereby improving insulin resistance and lipid metabolism as well as reducing the blood pressure. ${ }^{11,12}$ However, the effect of Telmisartan on the development of unfavorable LV remodeling after MI has not been investigated.

The aim of this study was to determine whether treatment with Telmisartan could improve the LV dysfunction, LV dilatation, hypertrophy of noninfarcted myocardium, and inflammation in an animal model of MI. The involvement of PPAR $-\gamma$ in the progression of unfavorable LV remodeling after MI was also examined.

\section{SUBJECTS AND METHODS Animals}

Male Sprague-Dawley rats, (8-10 weeks old and weighing 200-250 g) were purchased from Japan Clea (Tokyo, Japan). All experiments were performed in accordance with the Guide for the Care and Use of Laboratory Animals (NIH Publication No. 85-23, revised 1996) and were approved by the Animal Research Committee of Tokyo Medical and Dental University.

\section{Surgical Procedure}

Rats were anesthetized with intraperitoneal pentobarbital sodium $(40-60 \mathrm{mg} / \mathrm{kg})$ and intubated with a polyethylene tube for ventilation (SN-480-7, Shinano, Tokyo, Japan). After thoracotomy was performed at the fourth intercostals space, the pericardium was gently opened to expose the heart, and myocardial ischemia was produced by ligation of the left anterior descending artery (LAD). Then the chest was closed, and the animals were allowed to recover in a warm, clean cage. ${ }^{13}$ Rats that died within $24 \mathrm{~h}$ of the operation were excluded from this study.

\section{Treatment}

Telmisartan was kindly provided by Boehringer Ingelheim Losartan, an ARB, was purchased from LKT Laboratories (St Paul, MN, USA) and specific PPAR- $\gamma$ antagonist GW9662 (2-chloro-5-nitrobenzanilide) was purchased from WAKO Chemicals (Tokyo, Japan). Rats were given chow that contained Telmisartan at $1 \mathrm{mg} / \mathrm{kg} /$ day (Telmisartan group) or Losartan at $1 \mathrm{mg} / \mathrm{kg} /$ day (Losartan group), or standard chow (Vehicle group) for 28 days from $24 \mathrm{~h}$ after the operation (day 0). In the Telmisartan plus GW9662 (TEL + GW9662) group, rats were injected intraperitoneally with GW9662 at $1 \mathrm{mg} / \mathrm{kg}$ twice daily for 28 days. In the sham control group, rats underwent a sham operation (thoracotomy and LAD isolation without ligation) and were fed standard chow for 28 days (Sham group).

\section{Blood Pressure Measurement}

Blood pressure was measured in conscious rats by using a tail-cuff system (BP-98A; Softron, Yokohama, Japan). It was measured just before surgery and was also measured at 7, 14, 21 , and 28 days after induction of MI.

\section{Echocardiography}

Transthoracic echocardiography was performed at 28 days after the operation. The animals were lightly anesthetized with pentobarbital sodium. An ultrasound apparatus with a $15-\mathrm{MHz}$ annular array transducer was used to obtain twodimensional targeted M-mode echocardiogram in the shortaxis view of the left ventricle at the level of the papillary muscles. The LV end-diastolic dimension (LVEDD), LV endsystolic dimension, LV posterior wall (LVPW) thickness, and fractional shortening ratio determined, and the LV ejection fraction (LVEF) was calculated by the area-length method. As an index of LV diastolic function, the ratio of the peak velocity of the early (E) to late (A) filling waves was determined from Doppler images of mitral flow obtained in the apical four-chamber view, as described previously. ${ }^{13}$ Each value was the average of measurements over three consecutive beats, and data were obtained by blinded observers.

\section{Measurement of the Plasma Concentration of Brain Natriuretic Peptide}

After echocardiography, a blood sample was obtained from the inferior vena cava and the plasma concentration of brain natriuretic peptide (BNP) was determined by enzyme-linked immunosorbent assay (ELISA) (Angiopharm, O'Fallon, MO, USA) according to the manufacturer's instructions.

\section{Histological Examination}

All rats were killed after the experiment and the right and left ventricles were dissected and weighed. Then sections $(5 \mu \mathrm{m}$ thick) were obtained from the left ventricle for staining with Mallory-Azan stain and the stained area was defined as the infarcted area. The infarct size was calculated as the total circumference of infracted myocardium divided by the total LV circumference. The area stained blue in the noninfarcted region (at the mid-portion of the LV septum) was measured to evaluate the area of myocardial fibrosis using computerassisted analysis (Scion Image $\beta 4.0 .2$ ) at a magnification of $\times 200$ (ref. 14). Myocyte cross-sectional area was measured from images captured from wheat germ agglutinin (WGA) stained sections. Proper cross-sections were determined as having nearly circular capillary profiles and circular to oval myocyte sections. The outline of 100 myocytes was traced in each section. ${ }^{15}$

\section{Evaluation of Apoptosis in Tissue Sections}

DNA fragmentation was detected in situ using terminal deoxynucleotidyl transferase dUTP nick end labeling (TUNEL) as described previously. ${ }^{15}$ Nuclear density was determined by counting $4^{\prime}, 6$-diamidino-2-phenylindolestained nuclei in 20 different fields for each animal. 


\section{Immunohistochemistry}

Cardiac specimens were frozen in optimum cutting temperature compound (Sakura Finetek, Tokyo, Japan) and cut into $5 \mu \mathrm{m}$ thick sections. Each section was incubated overnight at $4{ }^{\circ} \mathrm{C}$ with the primary antibody for ED1 (Serotec, Oxford, UK) and then at room temperature for $30 \mathrm{~min}$ with Histofine Simple Stain Rat (Nichirei, Tokyo, Japan), followed by reaction with AEC matrix solution (Nichirei).

\section{Extraction of Proteins from Heart Sections}

Heart sections were homogenized in an extraction buffer containing $50 \mathrm{mM}$ Tris- $\mathrm{HCl}$ ( $\mathrm{pH} 7.5), 150 \mathrm{mM} \mathrm{NaCl}, 1 \%$ Triton X-100, $2 \mathrm{mM}$ EGTA, $10 \mathrm{mM}$ EDTA, $100 \mathrm{mM} \mathrm{NaF}$, $1 \mathrm{mM} \mathrm{Na}{ }_{4} \mathrm{P}_{2} \mathrm{O}_{7}, 2 \mathrm{mM} \mathrm{Na} \mathrm{VO}_{4}, 100 \mu \mathrm{g} / \mathrm{ml}$ PMSF, and protease inhibitor. After centrifugation, the second supernatant layer was collected for the analysis of cardiac proteins.

\section{Western Blot Analysis}

Protein concentrations were measured with a BCA protein assay (Thermo Scientific, Rockford, IL, USA) to equalize the amount of total protein. SDS-polyacrylamide gel electrophoresis was performed with $10 \%$ gels, followed by transfer to polyvinilidene difluoride membranes by the semi-dry electrotransfer method. Then membranes were incubated with the primary antibodies overnight at $4{ }^{\circ} \mathrm{C}$, incubated for $2 \mathrm{~h}$ with the secondary antibody and developed with enhanced chemiluminescence reagent (GE Healthcare, Waukesha, WI, USA). Antibodies for PPAR- $\gamma$, TGF- $\beta 1$, Bax, $\mathrm{Bcl}-2$, and cleaved caspase- 3 were purchased from Santa Cruz Biotechnology (Santa Cruz, CA, USA).

\section{Gelatin Zymography}

The LV septum was harvested at 7 days after induction of MI, washed with cold PBS, and snap frozen in liquid nitrogen. For extraction of proteins, tissues were minced into $1 \mathrm{~mm}^{3}$ pieces and incubated with $0.5 \%$ Triton X-100 in PBS containing $0.01 \%$ sodium azide. After centrifugation, the supernatants were collected. MMP activity was measured by in-gel zymography with gelatin $(1 \mathrm{mg} / \mathrm{ml}$, type A from porcine skin; Sigma Chemical, St Louis, MO, USA) as the substrate, as described previously, ${ }^{14}$ with enzyme activity attributable to MMP-9 and MMP-2 being visualized as clear bands against a blue background. Recombinant human MMP-9 and MMP-2 (Biomol, Plymouth Meeting, PA, USA) were included in the gels as standards. MMP activity was quantified by densitometery.

\section{Tissue Inhibitor of Metalloproteinase-1 (TIMP-1) Activity Assay}

To analyze TIMP-1 activity, TIMP-1 Biotrak ${ }^{\text {TM }}$ ELISA was performed according to the manufacturer's instructions (GE Healthcare). Briefly, TIMP standards and samples (appropriately diluted) were added to the ELISA plates and incubated at $20-27^{\circ} \mathrm{C}$ for $1-2 \mathrm{~h}$. Wells were then aspirated and washed with wash buffer; horseradish peroxidase conjugate was added and the plates were incubated as before. Following aspiration and washing, 3,3',5,5'-tetrabenzidine substrate was added to each well and the plates were re-incubated for $30 \mathrm{~min}$. The reaction was stopped by the addition of $1 \mathrm{M}$ sulfuric acid and absorbances were read, within $30 \mathrm{~min}$, using a spectrophotometer equipped with a $450 \mathrm{~nm}$ filter. Calculation of the relative amounts of TIMP-1 in the samples was made by comparison with the respective standard curves.

\section{Cell Culture}

Neonatal cardiomyocytes were isolated from 1- or 2-day-old Wistar rats, subjected to Percoll gradient centrifugation, and cultured as described previously. ${ }^{16,17}$ The cardiomyocytes were incubated at $37^{\circ} \mathrm{C}$ in Eagle's minimum essential medium (Sigma Chemical) supplemented with $5 \%$ calf serum (JRH Biosciences, Lenexa, KS, USA). Cardiac fibroblasts were isolated as described previously and were cultured in Dulbecco's modified Eagle's medium supplemented with $10 \%$ fetal bovine serum. ${ }^{18}$ Cells from the second to fourth passages were used for all experiments to exclude contamination by cardiomyocytes. After the growth medium was replaced with serum-free medium containing various concentrations of Telmisartan, Losartan, or a PPAR- $\gamma$ antagonist (GW9662), cells were exposed to hypoxic or normoxic conditions for $24 \mathrm{~h}$. To create hypoxia, the cells were placed in a Plexiglas chamber and incubated under water-saturated $95 \% \mathrm{~N}_{2} / 5 \% \mathrm{CO}_{2}$.

\section{PPAR- $\gamma$ Transcriptional Activity}

The nuclear fraction was isolated from cultured cardiomyocytes with a Nuclear Extract Kit (Chemicon International, Temecula, CA, USA) in accordance with the manufacturer's protocol, and the remaining cytoplasmic fraction was used for western blot analysis as described above. Activation of PPAR- $\gamma$ was assessed with the $\operatorname{TransAM}^{\circledR}$ ELISA-based kit (Active Motif, Rixensart, Belgium) according to the manufacturer's directions. In brief, nuclear extracts were incubated for $1 \mathrm{~h}$ in a 96-well plate coated with a consensus PPAR- $\gamma$ sequence. After washing the plate, the extracts were incubated with PPAR- $\gamma$ antibody for $1 \mathrm{~h}$, followed by incubation with a horseradish peroxidase-conjugated secondary antibody. PPAR- $\gamma$ activation was detected by a colorimetric assay and was quantified by spectrophotometry at $450 \mathrm{~nm}$ with a reference wavelength of $655 \mathrm{~nm}$. The PPAR- $\gamma$ transfected nuclear extract provided with the kit was used as the positive control.

\section{Real-Time RT-PCR Analysis}

Total RNA was extracted from cardiac fibroblast with TRIsure ${ }^{\circledR}$ reagent (Nippon Genetics, Tokyo, JAPAN) and subjected to quantitative RT-PCR analysis with primers specific for rat cDNAs encoding connective tissue growth factor (CTGF) and OPN. Sequence of primers for rat CTGF, OPN and glyceraldehyde-3-phosphate dehydrogenase (GAPDH) 
are as follows: MMP-2; 5'-GGCCATGCCATGGGGCTGGA-3' (forward), 5' $\quad$-CCAGTCTGATTTGATGCTTC-3' ${ }^{\prime}$ (reverse); MMP-9; 5'-TGTTCAGCAAGGGGCGTGTC-3' (forward), 5' AAACAGTCCAACAAGAAAGG-3' (reverse); TIMP-1; 5'-CT GGCATCCTCTTGTTGCTA-3' (forward), 5'-AGGGATCTCC AGGTGCACAA-3' (reverse); CTGF; 5'-AAGAAGACTCAGCC AGACC-3' (forward), 5' (reverse); OPN; 5'-CTCGGAGGAGAAGGCGCATTA-3 ${ }^{\prime}$ (forward), $\quad 5^{\prime}$-CCATCGTCATCGTCGTCGTGA-3' (reverse); GAPDH; 5'-GCCTTCTCCATGGTGGTGAA-3' (forward), $5^{\prime}$-GGTCGGTGTGAACGGATTTG-3' (reverse). GAPDH mRNA was used as an internal standard.

\section{Statistical Analysis}

All statistical analyses were performed with Prism for Macintosh software (Version 4; Graphpad, San Diego, CA, USA). Results are presented as the mean \pm s.e.m. All data were analyzed by analysis of variance followed by Scheffe's test for multiple comparisons. The Mann-Whitney $U$-test was used to compare survival rates between two groups and a $P$-value of $<0.05$ was considered significant.

\section{RESULTS}

\section{Mortality and Heart Failure after Induction of MI}

Approximately, $40 \%$ of the rats survived production of MI and were assigned to the Telmisartan group, the Losartan group, the TEL + GW9662 group, and the Vehicle group. The 28-day survival rate was significantly higher in the Telmisartan group than in the Losartan group, the TEL + GW9662 group, and the Vehicle group $(P<0.05)$ (Figure 1a). There was no significant difference in systolic blood pressure at 28 days after induction of MI (Figure 1b). Although there was no significant differences in infarct size (Figure 1c), the LV weight/body weight ratio was significantly lower in the Telmisartan group than in the Losartan group, the TEL + GW9662 group and the Vehicle group (Figure 2a). The lung weight/body weight ratio was also significantly lower in the Telmisartan group than in the Losartan group, the TEL + GW9662 group, and the Vehicle group (Figure 2a). Plasma BNP levels in each group are shown in Figure 2b. At 28 days after the induction of MI, plasma BNP level was significantly lower in the Telmisartan group compared with the Losartan group, the TEL + GW9662 group, and the Vehicle group $(P<0.05)$.
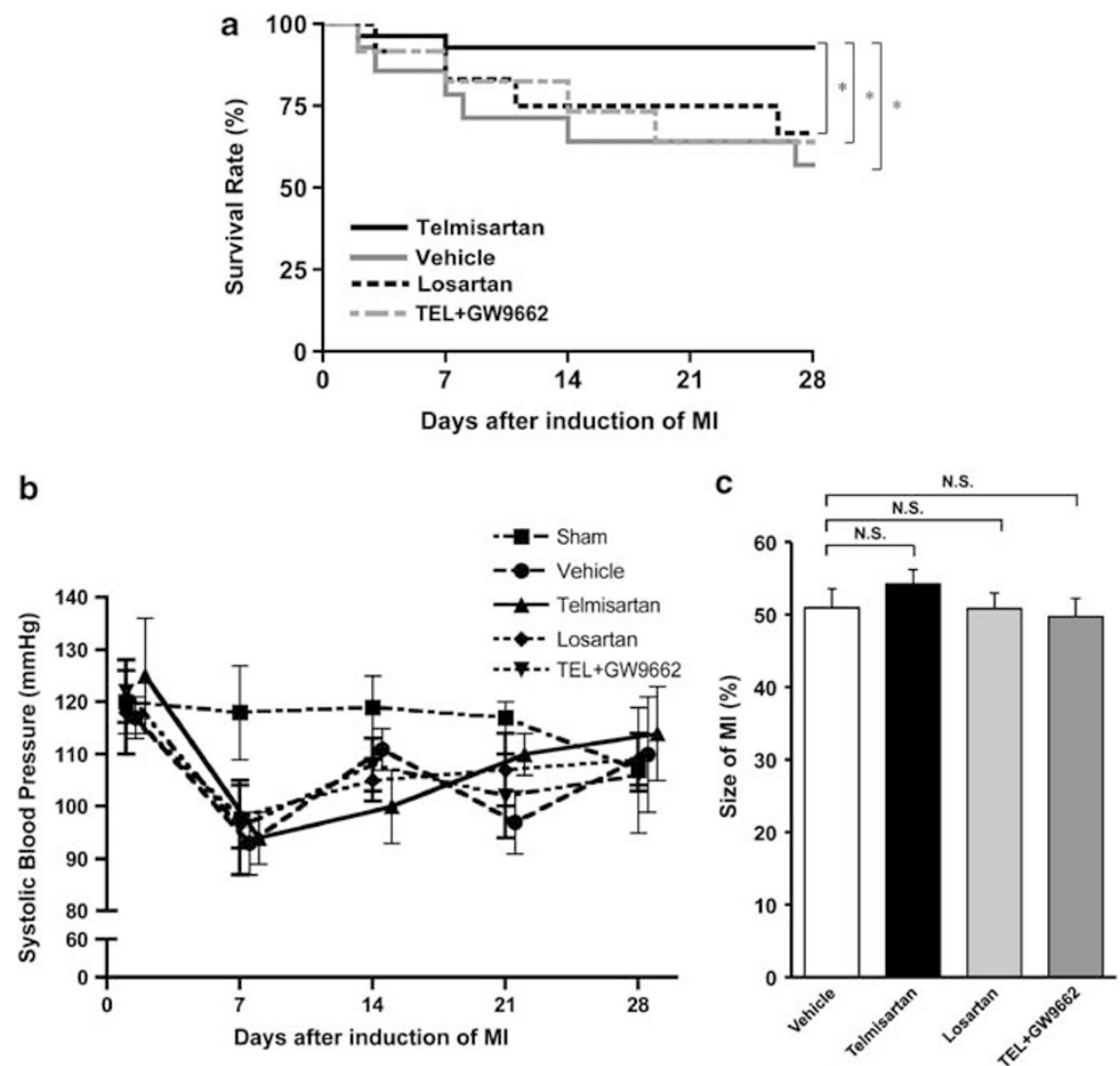

Figure 1 (a) Survival of rats with myocardial infarction (MI). At 28 days after induction of Ml, the survival rate of the Telmisartan group $(n=8)$ was significantly better than that of the Vehicle group $(n=14)$, the Losartan group $(n=12)$, and the Telmisartan + GW9662 (TEL + GW9662) group ( $n=12$ ). ${ }^{\star} P<0.05$ vs Telmisartan. (b) Systolic blood pressure just before the surgical procedure (pre-MI) and 7, 14, 21, and 28 days after induction of MI. No significant differences in systolic blood pressure were observed between the Telmisartan group, the Losartan group, the TEL + GW9662 group, and the Vehicle group; $n=6$ per group. (c) The size of MI was determined as described in Subjects and methods section. 


\section{Echocardiographic Findings}

LVEDD was smaller, while the LVEF was greater in the Telmisartan group compared with the Losartan group, the TEL + GW9662 group, and the Vehicle group, indicating that LV systolic function was significantly improved by treatment with Telmisartan (Figures $3 \mathrm{a}$ and $\mathrm{b}$ ). Improvement of hypertrophy in the noninfarcted myocardium, suggested by a smaller LVPW thickness, was observed in the Telmisartan group (Figure 3c). Moreover, significant improvement of LV diastolic function was observed in the Telmisartan group by pulse-wave Doppler recording of mitral inflow, which showed a smaller E/A ratio than that of the Losartan group, the TEL + GW9662 group, and the Vehicle group (Figure 3d).

\section{Histological Findings}

The extent of interstitial fibrosis in the noninfarcted area (assessed by Mallory-Azan staining) was significantly smaller in the Telmisartan group compared with the Losartan group, the TEL + GW9662 group, and the Vehicle group (Figure 4a). Immunohistochemical staining for ED1 showed significantly less accumulation of ED1-positive macrophages in the non- infarcted region of the Telmisartan group compared with the Losartan group, the TEL + GW9662 group, and the Vehicle group (Figure $4 \mathrm{~b}$ ). These results suggest that cardiac fibrosis and inflammatory cell infiltration of the noninfarcted area were prevented by treatment with Telmisartan and this effect was abrogated by PPAR- $\gamma$ inhibition. The size of cardiomyocyte diameter in the noninfarcted area assessed by WGA staining was significantly smaller in the Telmisartan group compared with the Losartan group, the TEL + GW9662 group and the Vehicle group (Figure 4c). This finding indicated that cardiac hypertrophy of the noninfarcted area was prevented by treatment with Telmisartan, and that this effect was mediated by PPAR- $\gamma$. LV remodeling is associated with increased apoptosis in the myocardium. ${ }^{19}$ To determine the extent of apoptosis, TUNEL-positive nuclei were counted in the remodeling area. In the Vehicle group, the number of TUNEL-positive cardiomyocytes was significantly greater in rats subjected to MI than in sham-operated rats, suggesting that apoptosis is enhanced during cardiac remodeling. Although the number of TUNEL-positive cardiomyocytes in the remodeling area was also higher in the Telmisartan group
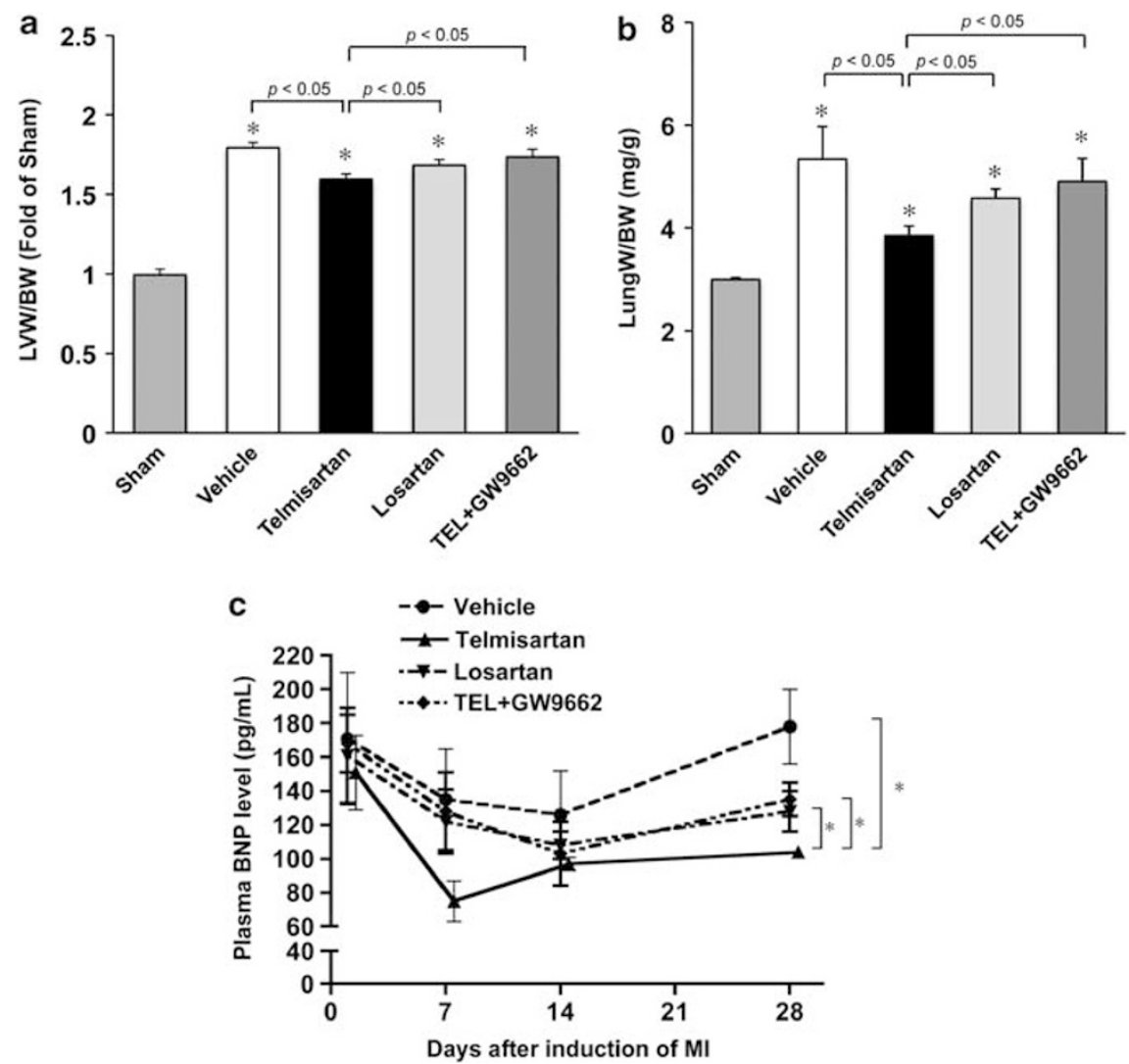

Figure 2 (a) Left ventricular weight/body weight (LVW/BW) was measured in the Sham group $(n=10)$, the Vehicle group ( $n=14)$, the Telmisartan group $(n=8)$, the Losartan group $(n=12)$, and the TEL + GW9662 group $(n=12) .{ }^{\star} P<0.05$ vs Sham. (b), Lung weight/body weight (lungW/BW) was measured in the Sham group $(n=10)$, the Vehicle group $(n=14)$, the Telmisartan group $(n=8)$, the Losartan group $(n=12)$, and the TEL + GW9662 group $(n=12)$. ${ }^{\star} P<0.05$ vs Sham. (c) Plasma BNP levels. BNP was significantly decreased at 28 days after induction of Ml in the Telmisartan group ( $\left.n=5\right)$ compared with the Vehicle group $(n=5)$, the Losartan group $(n=5)$, and the TEL + GW9662 group $(n=5) .{ }^{*} P<0.05$ vs Telmisartan. BNP, brain natriuretic peptide; MI, myocardial infarction. 

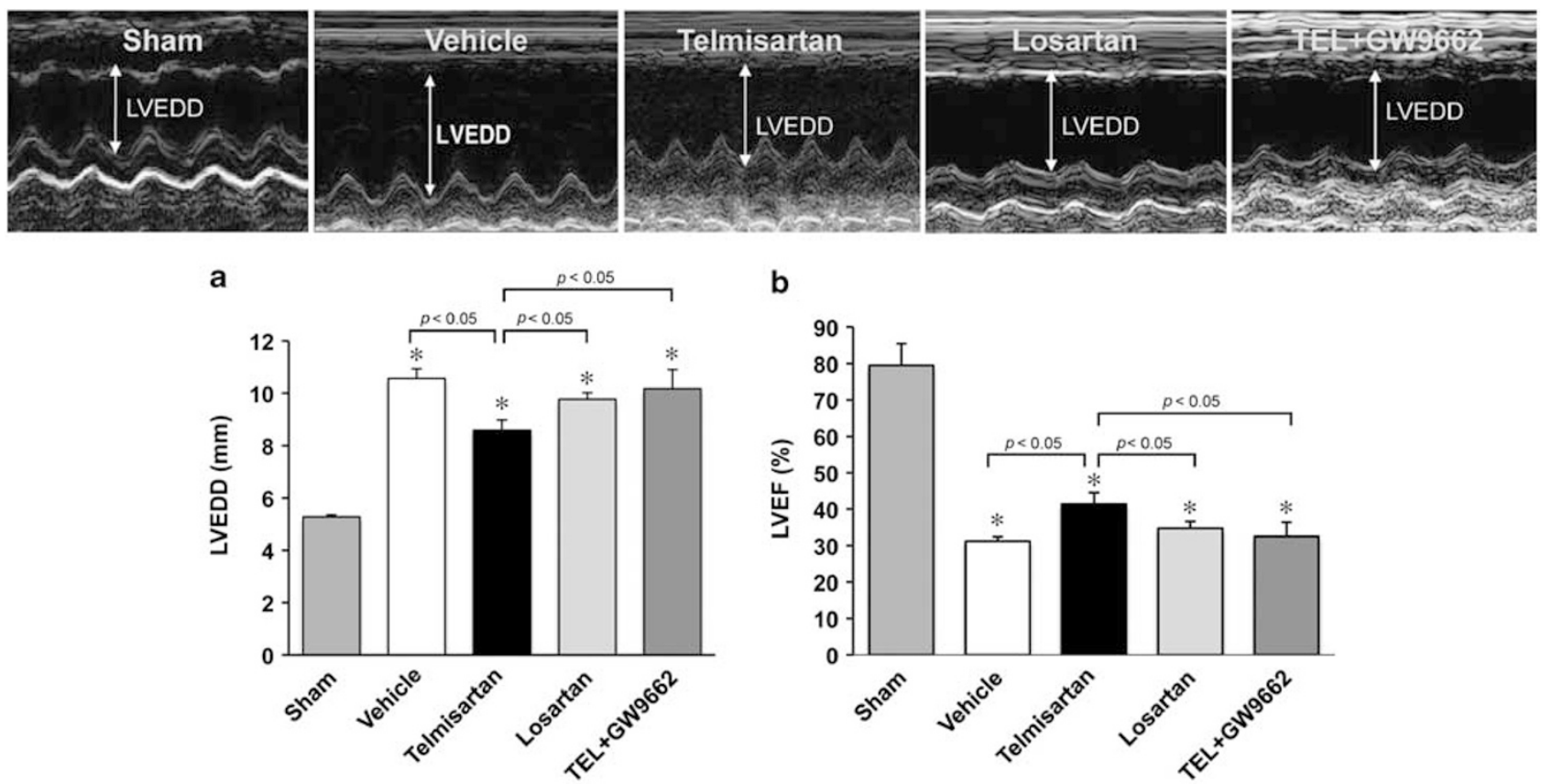

b
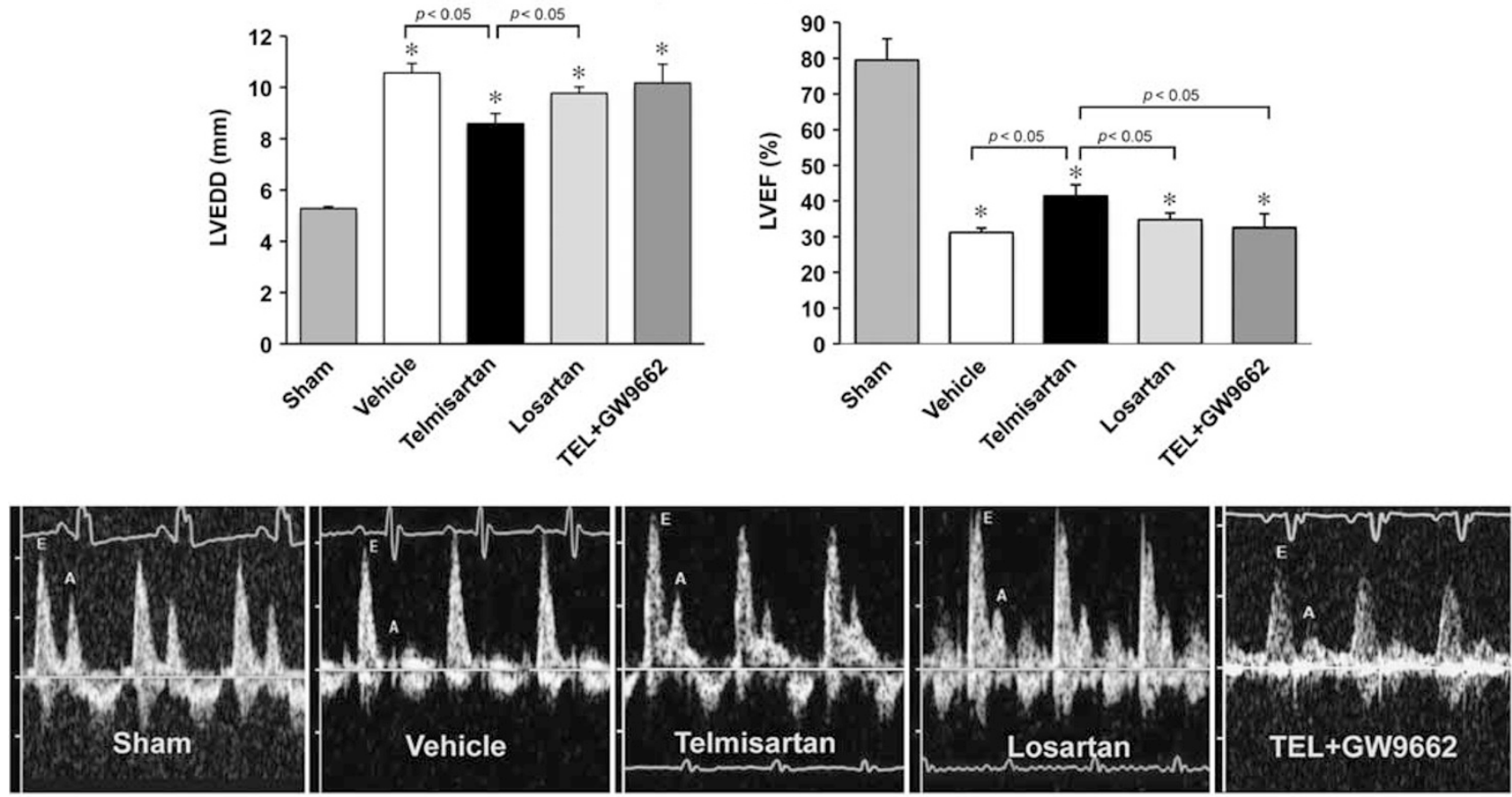

C

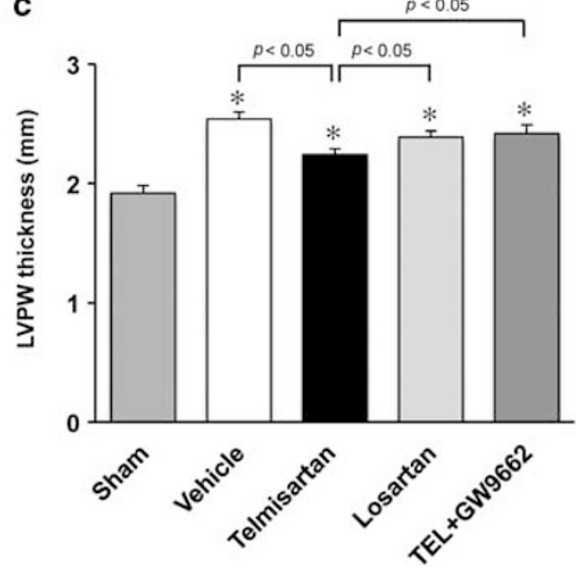

d

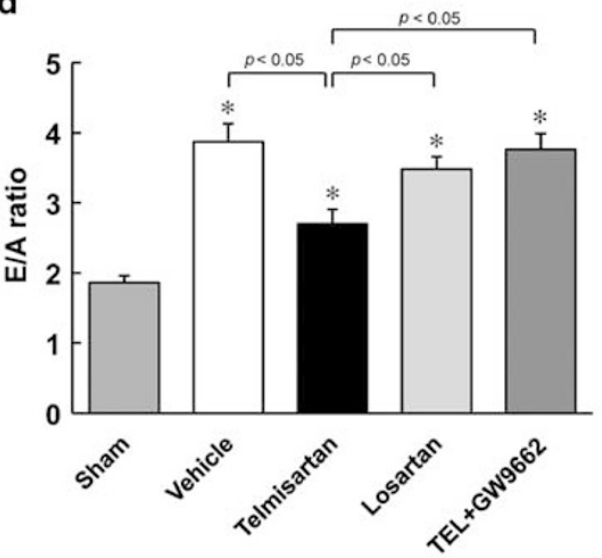

Figure 3 (a) Left panel: representative M-mode echocardiograms from the Sham group, the Vehicle group, the Telmisartan group, the Losartan group, and the TEL + GW9662 group at 28 days after induction of myocardial infarction. Right panel: quantitative analysis of left ventricular end-diastolic dimension (LVEDD) evaluated by the area-length method. ${ }^{*} P<0.05$ vs Sham. (b) Quantitative analysis of left ventricular ejection fraction (LVEF) evaluated by the arealength method. ${ }^{*} P<0.05$ vs Sham. (c) Quantitative analysis of left ventricular posterior wall (LVPW) thickness evaluated by the area-length method. ${ }^{\star} P<0.05$ vs Sham. (d) Transmitral flow velocity was measured in the Sham, Vehicle, Telmisartan, Losartan, and TEL + GW9662 groups (left panel: representative pictures) and the ratio of the early to late filling waves (E/A ratio) was calculated (lower panel). E, Early filling velocity; $A$, late filling velocity. ${ }^{\star} P<0.05$ vs Sham; $n=6$ per group.

than in sham-operated rats, the number of TUNEL-positive cardiomyocytes in the remodeling area was significantly less in the Telmisartan group compared with the Losartan group, the TEL + GW9662 group, and the Vehicle group (Figure 4d). We examined the expression of Bax, a proapoptotic protein, and Bcl-2, an anti-apoptotic protein, expression in the 

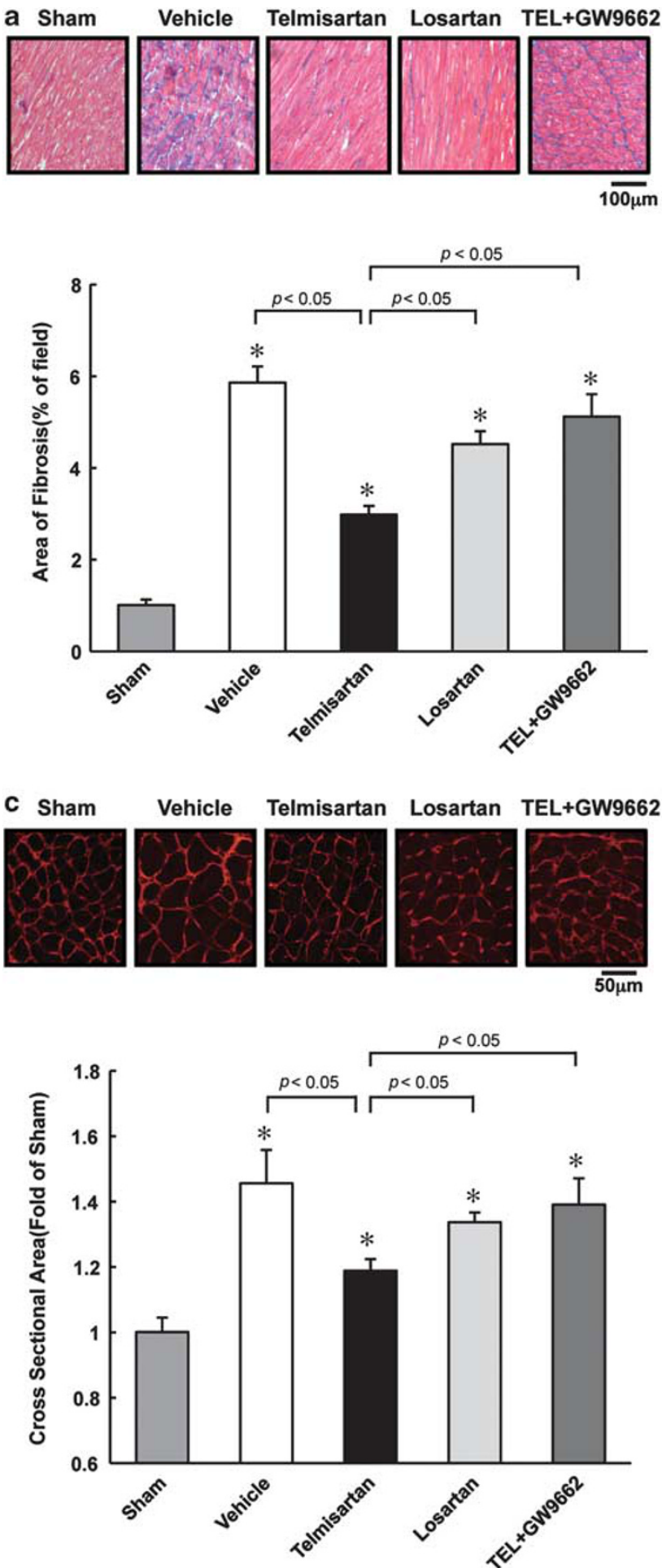

d

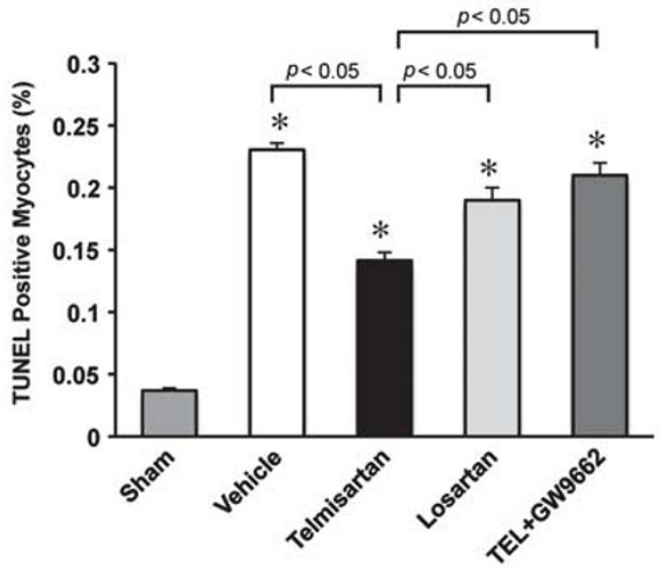

e
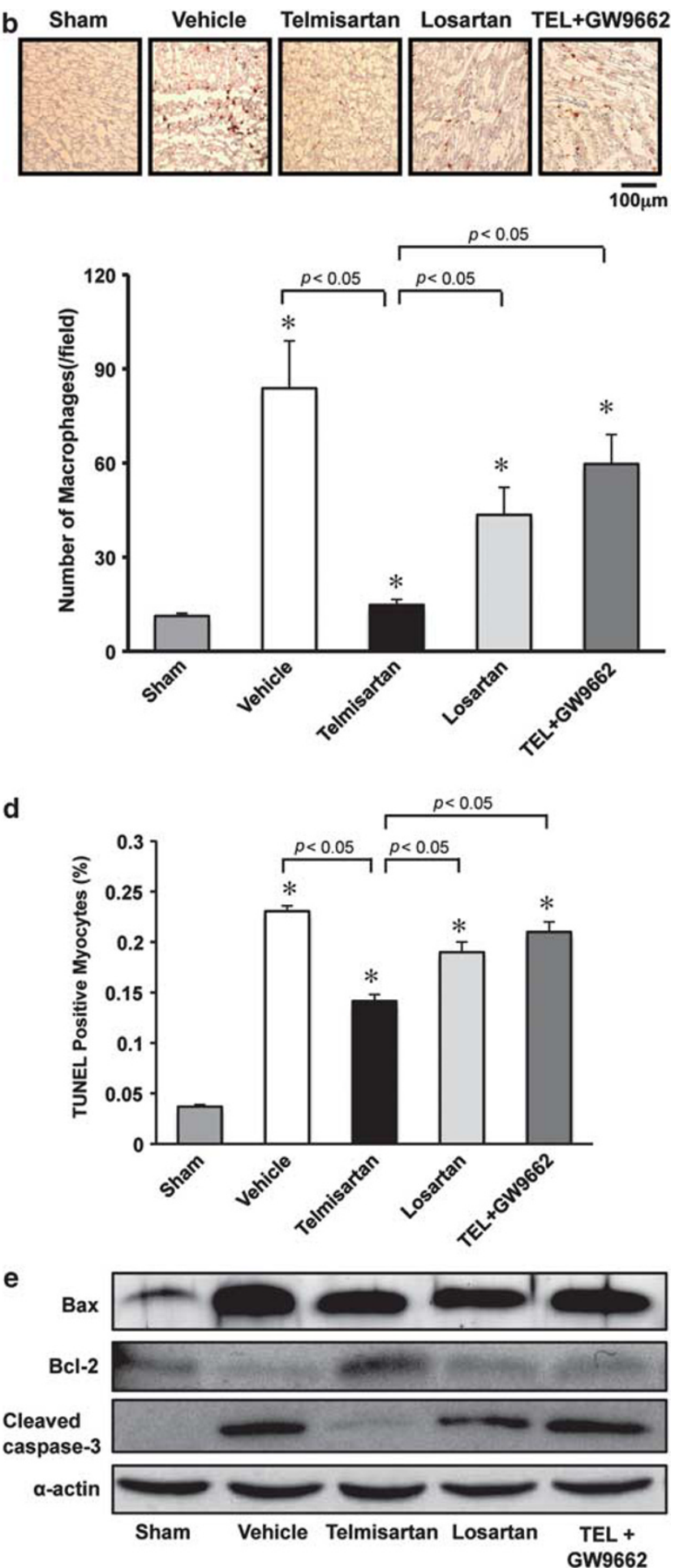

Figure 4 (a) Upper panel: representative images showing Mallory-Azan staining of noninfarcted myocardium from the Sham group, the Vehicle group, the Telmisartan group, the Losartan group, and the TEL + GW9662 group. Lower panel: the area of cardiac fibrosis in the noninfarcted region is presented as a percentage of the field. (b) Upper panel: representative images that show immunostaining of noninfarcted myocardium from the Sham group, the Vehicle group, the Telmisartan group, the Losartan group, and the TEL + GW9662 group. Lower panel: number of infiltrating macrophages in each section of noninfarcted myocardium. (c) Upper panel: representative images showing wheat germ agglutinin staining of noninfarcted myocardium from the Sham group, the Vehicle group, the Telmisartan group, the Losartan group, and the TEL + GW9662 group. Lower panel: quantitative comparison of the crosssectional diameter of cardiomyocytes. (d) The extent of apoptosis was determined by the number of TUNEL-positive myocytes divided by the number of 4',6-diamidino-2-phenylindole (DAPI)-positive nuclei. ${ }^{\star} P<0.05$ vs Sham; $n=6$ in each group. (e) Representative western blots of Bax, Bcl-2, and cleaved caspase-3 in the noninfarcted myocardium. 
a

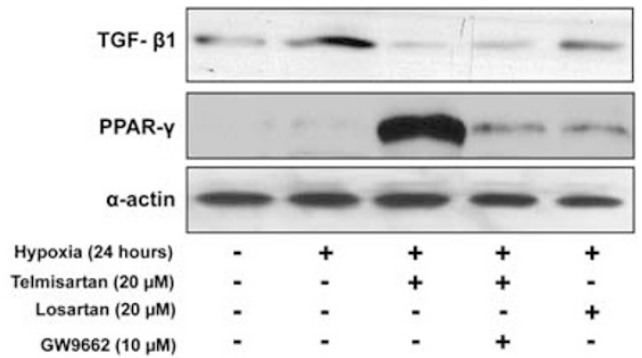

b

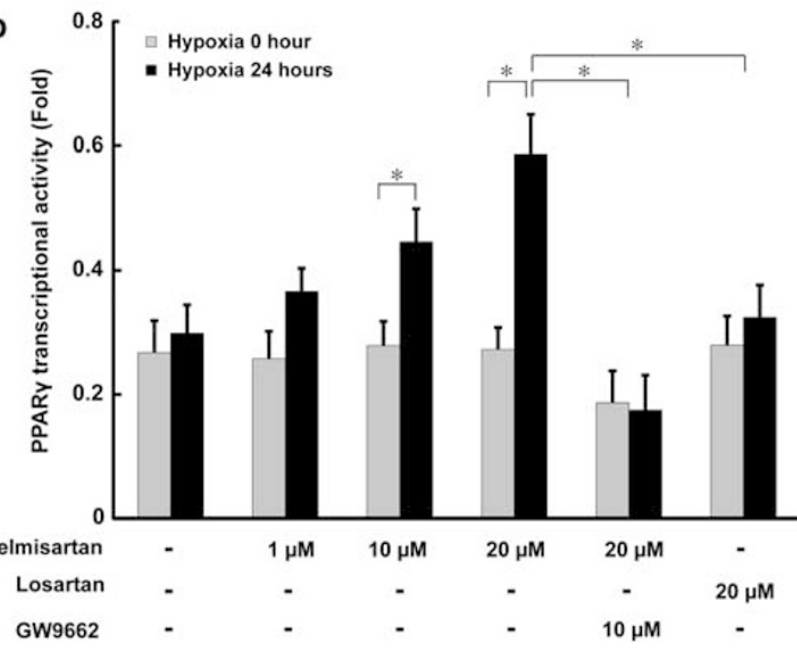

Figure 5 (a) Representative western blots of TGF- $\beta 1$ and PPAR- $\gamma$ in neonatal rat cardiomyocytes cultured under hypoxic conditions. (b) Changes in PPAR- $\gamma$ transcriptional activity in neonatal rat cardiomyocytes cultured under hypoxic conditions. Relative PPAR- $\gamma$ transcriptional activity expressed as the fold increase in optical density compared with the positive control. ${ }^{\star} P<0.05 ; n=6$ per group.

remodeling area of myocardium by western blot analysis. Bax expression was significantly increased in the heart subjected to MI than in that of sham-operated rats. However, there was no significant change of Bax expression among MI-subjected groups. Bcl-2 expression was significantly increased in the Telmisartan group compared with the Losartan group, the TEL + GW9662 group, and the Vehicle group (Figure 4e). We also evaluated caspase activity as determined by cleaved caspase-3 levels by western blot analysis. Cleaved caspase-3 levels were significantly increased in hearts subjected to MI than in that of sham-operated rats. However, cleaved caspase3 levels were significantly decreased by treatment with Telmisartan, and not decreased in the Losartan group and the TEL + GW9662 group (Figure 4e). These results suggest that apoptosis of cardiomyocytes after MI was prevented by treatment with Telmisartan through Bcl-2 upregulation, and this effect was attenuated by PPAR- $\gamma$ inhibition.

\section{Western Blot Analysis and PPAR- $\gamma$ Transcriptional Activity Assay of Cultured Cardiomyocytes}

To mimic ischemia in vitro, neonatal rat cardiomyocytes were cultured under hypoxic conditions. We examined the level of TGF- $\beta 1$ and PPAR- $\gamma$ protein expression in the cultured cardiomyocytes by western blot analysis at $24 \mathrm{~h}$ after incubation.
TGF- $\beta 1$ expression was significantly increased under hypoxic conditions, and this increase was significantly inhibited by treatment with Telmisartan $(20 \mu \mathrm{M})$. The inhibitory effect of the ARB Losartan $(20 \mu \mathrm{M})$ on TGF- $\beta 1$ expression was less than Telmisartan. Treatment with GW9662 $(10 \mu \mathrm{M})$ did not inhibit the increase in TGF- $\beta 1$ expression (Figure 5a). PPAR- $\gamma$ expression was significantly increased by treatment with Telmisartan but not Losartan in cardiomyocytes under hypoxic condition. This increase was inhibited by addition of GW9662 (Figure 5a). PPAR- $\gamma$ transcriptional activity in cultured cardiomyocytes was also evaluated. After $12 \mathrm{~h}$ of incubation, PPAR- $\gamma$ activity was increased by Telmisartan in a dose-dependent manner. This increase was inhibited by the addition of GW9662. Unlike Telmisartan, Losartan did not increase PPAR- $\gamma$ transcriptional activity (Figure $5 b$ ).

\section{Activity of MMP-2/9 and TIMP-1, and the MRNA Expression Level of CTGF and OPN in Cardiac Fibroblasts}

Both MMP-2 and MMP-9 mRNA expression levels in cardiac fibroblasts cultured under hypoxic conditions were evaluated by real-time RT-PCR following various interventions. At $24 \mathrm{~h}$ of hypoxic incubation significantly increased both MMP-2 and MMP-9 mRNA expression in cardiac fibroblasts, and pretreatment with Telmisartan attenuated this increase in a dose-dependent manner. The decrease in mRNA expression of MMP-2 and MMP-9 after incubation with Telmisartan was blocked by the addition of GW9662. The decrease in mRNA expression of MMP-2 and MMP-9 by Losartan was less marked than that elicited by Telmisartan (Figure 6a). Next, MMP-2/9 activity in cardiac fibroblasts was evaluated. The gelatinase activity of MMP-2/9 in cardiac fibroblasts was increased after $24 \mathrm{~h}$ of hypoxic incubation, and this increased activity was inhibited by pretreatment with Telmisartan in a dose-dependent manner. The decrease in MMP-2/9 activity after incubation with Telmisartan was prevented by the addition of GW9662. Unlike Telmisartan, Losartan did not decrease MMP-2/9 activity (Figure 6b). We also evaluated TIMP-1 mRNA levels in cardiac fibroblasts cultured under hypoxic conditions. Hypoxia significantly increased TIMP-1 mRNA expression in cardiac fibroblasts, and pretreatment with Telmisartan enhanced this increase. The increase in mRNA expression of TIMP-1 after incubation with Telmisartan was inhibited by the addition of GW9662. Unlike Telmisartan, Losartan did not increase TIMP-1 expression (Figure 6c, left panel). The activity of TIMP-1 in cardiac fibroblasts was evaluated by TIMP-1 Biotrak ELISA assay, which is specific for free TIMP-1 and TIMP- 1 complexed with active forms of MMPs, but not TIMP-1 complexed with pro-MMPs. The activity of TIMP-1 in cardiac fibroblasts was increased by hypoxia and this increased activity was upregulated further by pretreatment with Telmisartan. The increase in TIMP-1 activity after incubation with Telmisartan was abrogated by the addition of GW9662. Contrary to Telmisartan, Losartan did not decrease TIMP-1 activity 
a

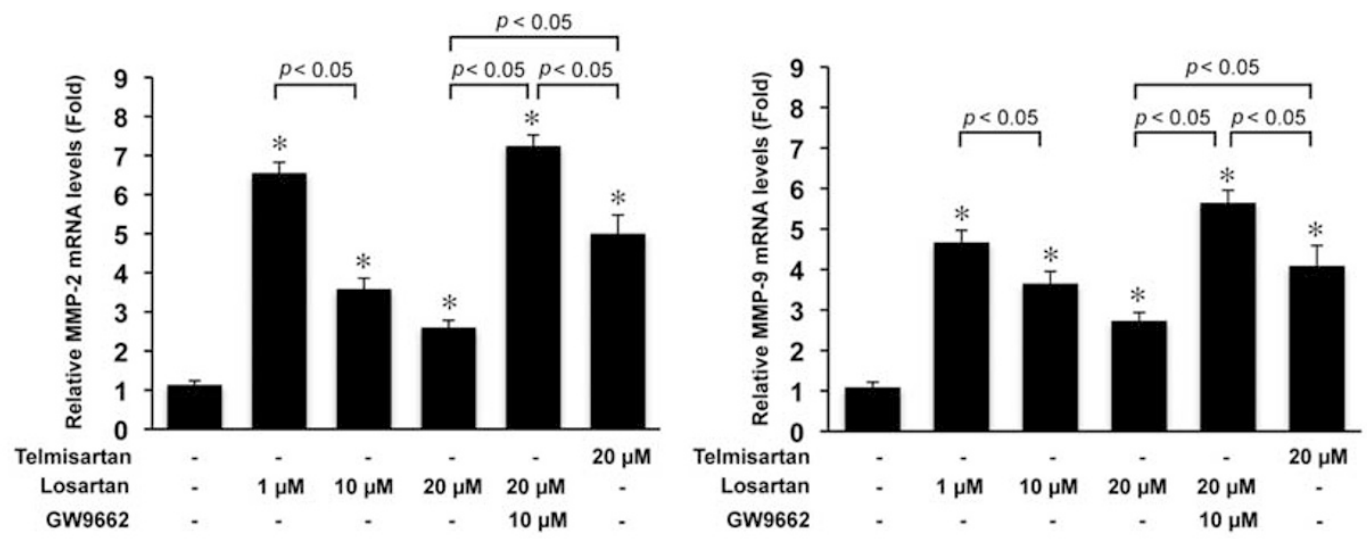

b

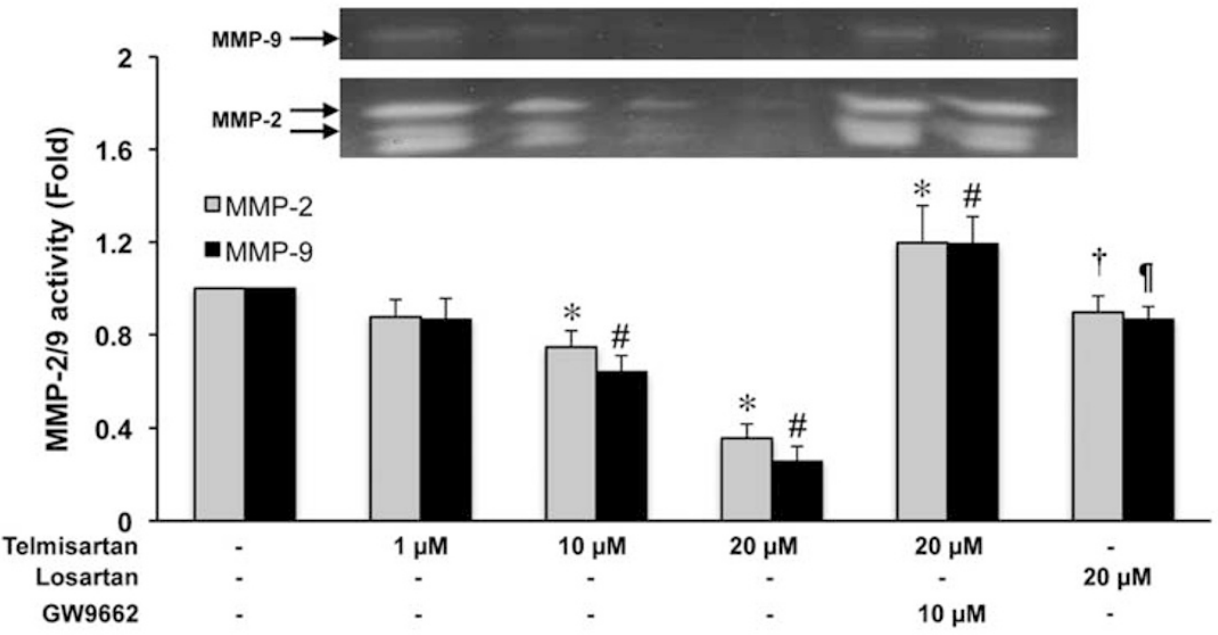

C

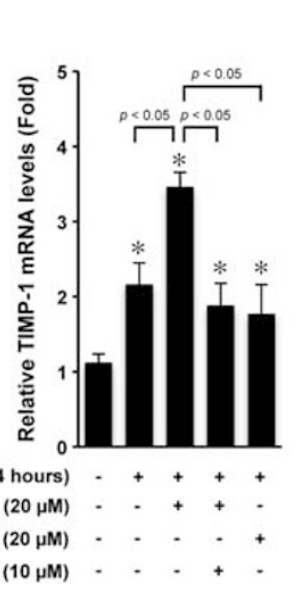

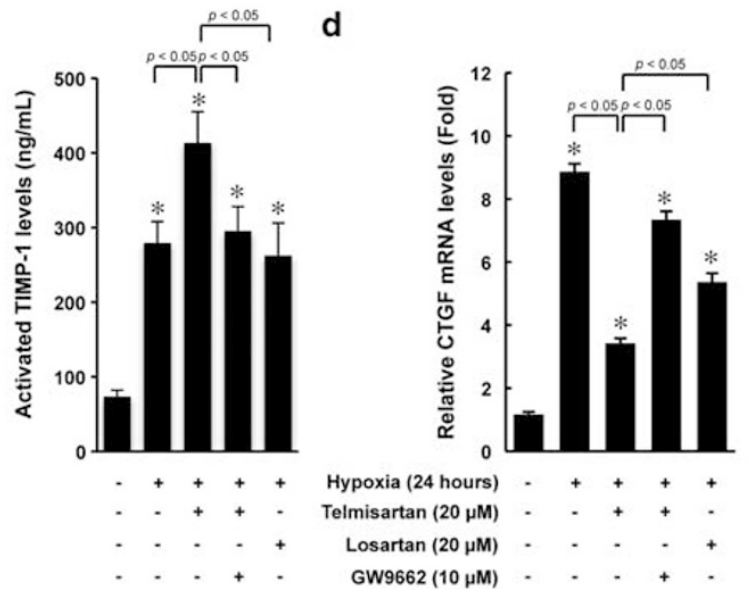

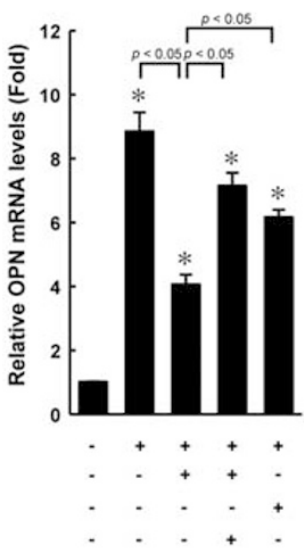

Figure 6 (a) Quantitative analyses of MMP-2 mRNA (left) and MMP-9 mRNA (right) expression in cardiac fibroblasts evaluated by MMP-2/GAPDH or MMP-9/ GAPDH expression ratio. ${ }^{*} P<0.05$ vs cardiac fibroblasts cultured under hypoxic conditions without treatment; $n=6$ per group. (b) Upper panel: representative image of in-gel gelatin zymography for MMP-2 and MMP-9 in cardiac fibroblasts. Lower panel: quantitative analysis of MMP-2 and MMP-9 activity in cardiac fibroblasts by in-gel gelatin zymography. ${ }^{\star} P<0.05$ vs cardiac fibroblasts cultured under hypoxic conditions without treatment (MMP-2), ${ }^{\#} P<0.05$ vs cardiac fibroblasts cultured under hypoxic conditions without treatment (MMP-9), $\uparrow P<0.05$ vs cardiac fibroblasts cultured under hypoxic conditions and treated with $20 \mu \mathrm{M}$ Telmisartan (MMP-2), ${ }^{\top} P<0.05$ vs cardiac fibroblasts cultured under hypoxic conditions and treated with $20 \mu \mathrm{M}$ Telmisartan (MMP-9). (c) Left panel: quantitative analysis of TIMP-1 mRNA expression in cardiac fibroblasts evaluated by TIMP-1/GAPDH expression ratio. Right panel: quantitative analysis of activated TIMP-1 protein level in cardiac fibroblasts assessed by TIMP-1 Biotrak ELISA. ${ }^{\star} P<0.05$ vs cardiac fibroblasts cultured under hypoxic conditions without treatment; $n=6$ per group. (d) Quantitative analysis of connective tissue growth factor (CTGF) mRNA (left) and OPN mRNA (right) expression in cardiac fibroblasts evaluated by CTGF/GAPDH or OPN/GAPDH expression ratio. ${ }^{\star} P<0.05 ; n=6$ per group. 

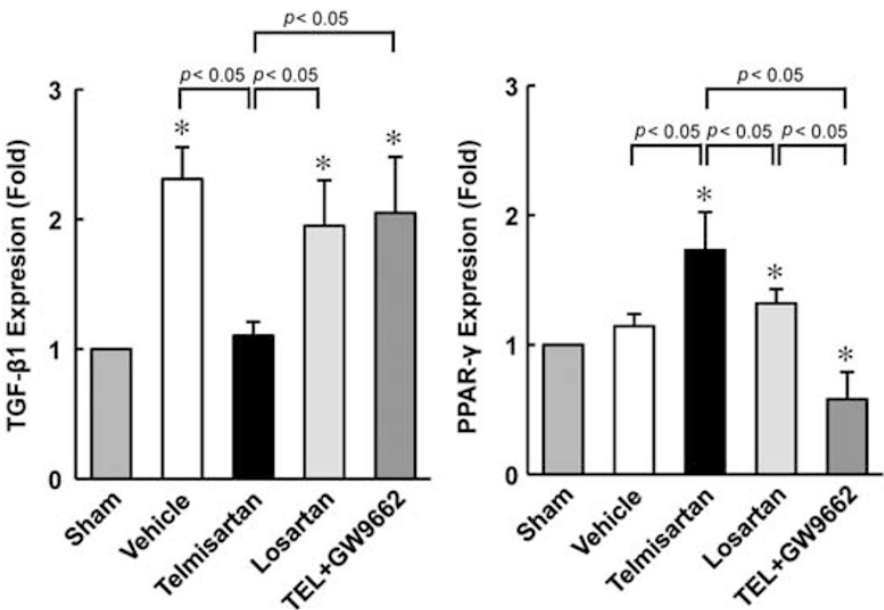

b
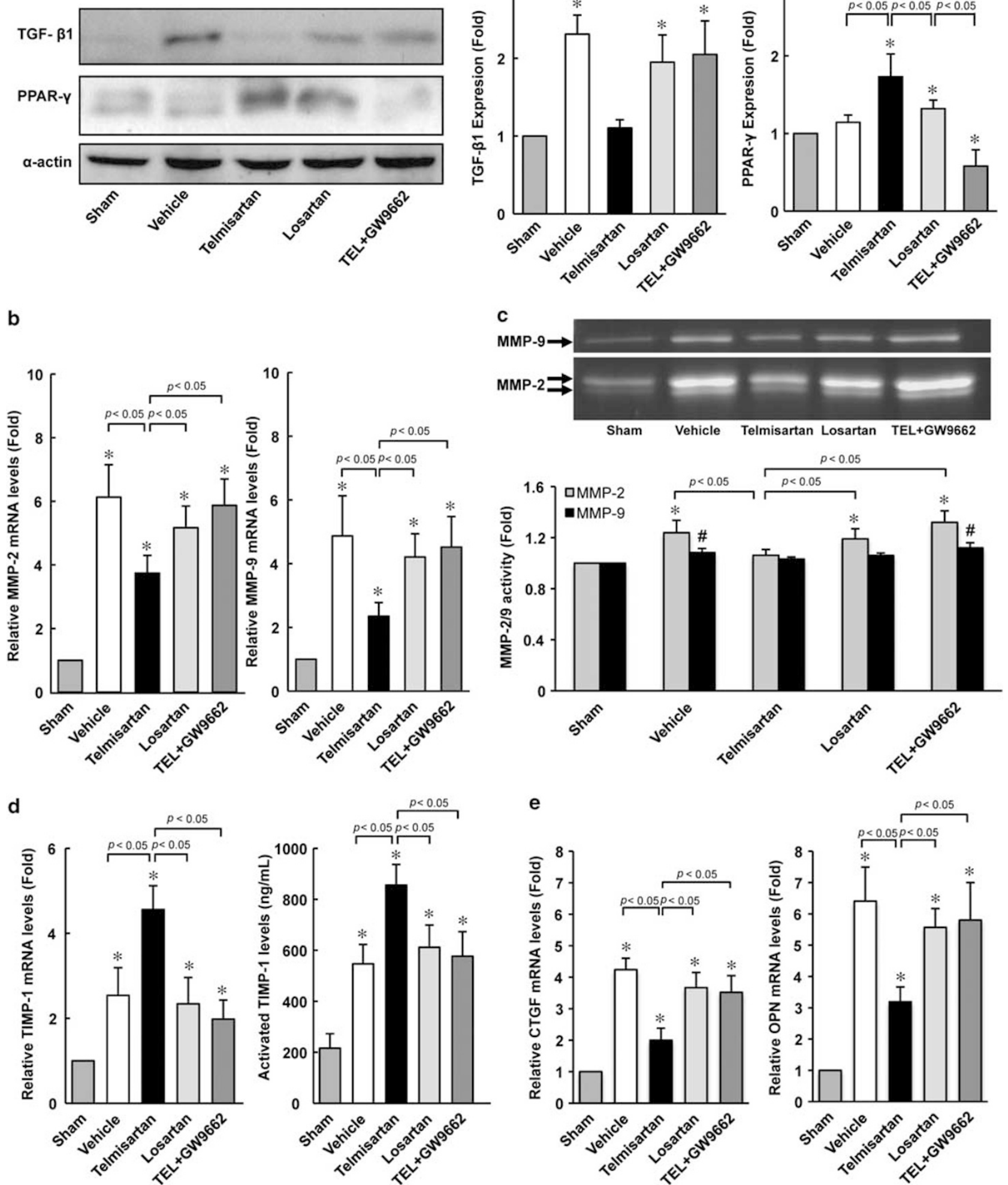

Figure 7 For caption see page 942. 
(Figure 6c, right panel). Then, the effect of hypoxic condition on CTGF and OPN mRNA expression in cardiac fibroblasts on was evaluated by real-time RT-PCR. CTGF is known as a downstream mediator of TGF- $\beta 1$ action on cardiac fibrosis. ${ }^{8}$ OPN is produced by cardiac fibroblasts in the heart through activation of cardiac AT1R and has a pivotal role in cardiac fibrosis. ${ }^{9,20}$ Hypoxic stimulation significantly increased both CTGF and OPN mRNA expression in cardiac fibroblasts, and pretreatment with Telmisartan attenuated this increase. The negative effect of Telmisartan on mRNA expression of CTGF and OPN was blocked by GW9662. The decrease in mRNA expression of CTGF and OPN by Losartan was less marked than that of Telmisartan (Figure 6d).

\section{Biochemical Analyses of Noninfarcted Myocardium}

We examined the level of TGF- $\beta 1$ and PPAR- $\gamma$ protein expression in the noninfarcted myocardium by western blot analysis at 7 days after induction of MI. TGF- $\beta 1$ expression was significantly reduced in the Telmisartan group compared with the Vehicle group. In both the Losartan group and the TEL + GW9662 group, TGF- $\beta 1$ expression slightly decreased compared with the Vehicle group (Figure 7a). PPAR- $\gamma$ expression was significantly increased in the Telmisartan group but not in the Losartan group. On the other hand, PPAR- $\gamma$ expression was significantly inhibited in the TEL + GW9662 group compared with the Vehicle group (Figure 7a). Next, both MMP-2 and MMP-9 mRNA expression levels in the noninfarcted myocardium were evaluated. Both MMP-2 and MMP-9 expression was significantly increased in the heart subjected to MI compared with that of sham-operated rats, and this increase was significantly attenuated in the Telmisartan group (Figure 7b). However, there was no significant difference of MMP-2 and MMP-9 expression in both the Losartan group and the TEL + GW9662 group compared with the Vehicle group (Figure $7 \mathrm{~b}$ ). In-gel gelatin zymography of the noninfarcted myocardium showed an increase in the gelatinase activity of both MMP-2 and MMP-9 in the Vehicle group compared with the Sham group. This increase in MMP activity was significantly smaller in the Telmisartan group (Figure 7c). Conversely, the increased activity of MMP-2 in the noninfarcted myocardium after MI was upregulated further in the TEL + GW9662 group. Unlike Telmisartan, Losartan did not decrease MMP-2/9 activity. We also evaluated TIMP-1 mRNA expression in the noninfarcted myocardium after MI. TIMP-1 expression was significantly increased in the Telmisartan group compared with the Vehicle group. However, there was no significant difference of TIMP-1 expression in noninfarcted myocardium among the Vehicle group, the Losartan group, and the TEL + GW9662 group (Figure $7 \mathrm{~d}$, left panel). The activity of TIMP-1 in noninfarcted myocardium was increased after MI and this increased activity was upregulated further in the Telmisartan group. Similar to TIMP-1 expression, there was no significant difference of TIMP-1 activity in noninfarcted myocardium among the Vehicle group, the Losartan group, and the TEL + GW9662 group (Figure 7d, right panel). We then examined the level of CTGF and OPN mRNA expression in the noninfarcted myocardium. Both CTGF and OPN mRNA expression was significantly reduced in the Telmisartan group compared with the Vehicle group (Figure 7e). Both CTGF and OPN mRNA expression was significantly increased in the heart subjected to MI compared with that of sham-operated rats, and this increase was significantly attenuated in the Telmisartan group. However, there was no significant difference of CTGF and OPN mRNA expression among the Vehicle group, the Losartan group, and the TEL + GW9662 group (Figure $7 \mathrm{e}$ ).

\section{DISCUSSION}

This study demonstrated that Telmisartan attenuates unfavorable LV remodeling after MI, as evidenced by a reduction of LV cavity size, inhibition of hypertrophy of the noninfarcted LV, and improvement of systolic and diastolic function. These beneficial changes were not associated with an effect on either blood pressure or infarct size, indicating that Telmisartan can improve unfavorable LV remodeling after MI via a 'blood pressure-independent effect'.

Although dilatation of the LV cavity is one of the compensatory responses of the failing heart after MI, excess dilatation results in LV dysfunction and leads to the development of heart failure. Prevention of unfavorable LV remodeling is important for improving the morbidity and mortality of patients after MI. The renin-Ang-aldosterone system (RAAS) has crucial role in the development of unfavorable LV remodeling. There is evidence that the RAAS not only induces the accumulation of collagen in the

Figure 7 (a) Upper panel: representative western blots of transforming growth factor- $\beta 1$ (TGF- $\beta 1$ ) and PPAR- $\gamma$ in the noninfarcted myocardium. Lower panel: relative expression of TGF- $\beta 1$ shown as a fold increase compared with the Sham group (left), and relative expression of PPAR- $\gamma$ shown as a fold increase compared with the Sham group (right). ${ }^{\star} P<0.05$ vs Sham; $n=6$ per group. (b) Quantitative analyses of MMP-2 mRNA (left) and MMP-9 mRNA (right) expression in noninfarcted myocardium evaluated by MMP-2/GAPDH or MMP-9/GAPDH expression ratio. ${ }^{\star} P<0.05$ vs Sham; $n=6$ per group. (c) Upper panel: representative gelatin in-gel zymographies of matrix metalloprotease-9 (MMP-9) and MMP-2 in the noninfarcted myocardium. Lower panel: relative expression of MMP-9 (left) and MMP-2 (right) shown as a fold increase compared with the Sham group. ${ }^{*} P<0.05$ vs Sham (MMP-2), ${ }^{\#} P<0.05$ vs Sham (MMP-9); $n=6$ per group. (d) Left panel: quantitative analysis of TIMP-1 mRNA expression in the noninfarcted myocardium evaluated by TIMP-1/GAPDH expression ratio. Right panel: quantitative analysis of activated TIMP-1 protein level in the noninfarcted myocardium assessed by TIMP-1 Biotrak ELISA. ${ }^{\star} P<0.05$ vs Sham; $n=6$ per group. (e) Quantitative analysis of connective tissue growth factor (CTGF) mRNA (left) and osteopontin (OPN) mRNA (right) expression in the noninfarcted myocardium evaluated by CTGF/GAPDH or OPN/GAPDH expression ratio. ${ }^{\star} P<0.05$ vs Sham; $n=6$ per group. 
infarcted heart, including the nonischemic area that causes myocardial stiffness, but also increases salt and water retention and total peripheral resistance, eventually leading to chronic heart failure. ${ }^{21}$ An experimental study showed that the expression and accumulation of collagen in the LV were significantly reduced by ARB therapy in MI rat model. ${ }^{22}$

Accumulating lines of evidence suggest that Losartan, an $\mathrm{ARB}$, has beneficial roles in inhibiting unfavorable LV remodeling after MI in experimental rat model. ${ }^{1,23,24}$ In this study, however, we demonstrated that treatment with Telmisartan significantly decreased the accumulation of macrophages, reduced cardiac fibrosis, and cardiomyocyte apoptosis, thereby attenuating unfavorable post-MI LV remodeling more effectively than treatment with Losartan. Furthermore, our current data reveal that co-administration of a PPAR- $\gamma$ antagonist (GW9662) with Telmisartan abolished its beneficial effect on LV remodeling after MI, suggesting that the advantage of Telmisartan over Losartan for the treatment of post-MI remodeling is due to PPAR- $\gamma$ agonistic activity. Several lines of evidence suggest that PPAR- $\gamma$ agonists have beneficial effects in patients with cardiovascular disease. ${ }^{25-27}$ The PROactive study, a large-scale randomized clinical trial, demonstrated that Pioglitazone, a full PPAR- $\gamma$ agonist, reduces the occurrence of all-cause mortality, non-fatal MI, and stroke in diabetic patients. ${ }^{28}$ Previous experimental investigations revealed that PPAR- $\gamma$ agonists can improve LV systolic and diastolic function by attenuating unfavorable LV remodeling in animal models of MI. ${ }^{3-5}$ Telmisartan inhibits Ang II type I receptor (AT1R) not only by direct blockade of AT1R but also by downregulation of AT1R expression through a PPAR- $\gamma$-mediated pathway, thereby inhibiting the RAAS more completely than other ARBs. ${ }^{29}$ Thus, Telmisartan effectively inhibits unfavorable LV remodeling via both AT1R blockade and an anti-inflammatory effect mediated by PPAR- $\gamma$ activation. Additionally, because there are unfavorable effects of full PPAR- $\gamma$ agonists such as liability to cause edema, a partial PPAR- $\gamma$ agonist such as Telmisartan may be more advantageous than full PPAR- $\gamma$ agonists to prevent heart failure after MI.

It is possible that the reduction of myocardial fibrosis in the noninfarcted regions contributed to improvement of LV relaxation and/or elasticity, and accumulating lines of evidence suggest that cardiac fibroblasts have an important role in post-MI adverse LV remodeling and in the development of cardiac failure via the production of matrix proteins that accumulate in the extracellular space. ${ }^{30-32}$ Recent studies using MMP knockout mice or administration of MMP inhibitors have provided insights into the involvement of particular MMP species in unfavorable LV remodeling after MI. ${ }^{33-36}$ In this study, the area of myocardial fibrosis, TGF- $\beta 1$ protein level, CTGF and OPN mRNA levels, and the activity of MMP-2 and MMP-9 were all increased in the noninfarcted myocardium 7 days after induction of MI compared with sham-operated rats. Importantly, Telmisartan ameliorated these unfavorable changes. PPAR- $\gamma$ agonists inhibit TGF- $\beta 1$ -
CTGF signaling, OPN expression, and the activity of MMP-2 and MMP-9. ${ }^{6,7,37,38}$ On the other hand, ARBs usually inhibit TGF- $\beta 1-C T G F$ signaling and OPN activity, but do not affect MMP-2 or MMP-9 activity. ${ }^{39-42}$ Thus, Telmisartan is a unique ARB that possesses potent anti-fibrotic activity via inhibition of MMP activation, OPN expression and TGF- $\beta 1$ CTGF signaling.

In conclusion, this study suggests that Telmisartan has a beneficial effect on post-infarct LV remodeling and dysfunction; therefore, it may be an effective therapy for preventing heart failure after MI.

\section{ACKNOWLEDGEMENTS}

We thank Dominic P Del Re for critical reading of the paper. This study was supported by a Grant-in-Aid for Scientific Research from the Ministry of Education, Science and Culture of Japan, a Research Grant for Diseases from the Ministry of Health and Welfare of Japan, and a Grant from the Japan Cardiovascular Research Foundation.

\section{DISCLOSURE/CONFLICT OF INTEREST}

The authors declare no conflict of interest.

1. Schieffer B, Wirger A, Meybrunn $M$, et al. Comparative effects of chronic angiotensin-converting enzyme inhibition and angiotensin II type 1 receptor blockade on cardiac remodeling after myocardial infarction in the rat. Circulation 1994;89:2273-2282.

2. Takahashi T, Anzai T, Yoshikawa T, et al. Angiotensin receptor blockade improves myocardial beta-adrenergic receptor signaling in postinfarction left ventricular remodeling: a possible link between beta-adrenergic receptor kinase-1 and protein kinase $C$ epsilon isoform. J Am Coll Cardiol 2004;43:125-132.

3. Lygate CA, Hulbert $\mathrm{K}$, Monfared $\mathrm{M}$, et al. The PPARgamma-activator rosiglitazone does not alter remodeling but increases mortality in rats post-myocardial infarction. Cardiovasc Res 2003;58:632-637.

4. Shiomi T, Tsutsui H, Hayashidani $\mathrm{S}$, et al. Pioglitazone, a peroxisome proliferator-activated receptor-gamma agonist, attenuates left ventricular remodeling and failure after experimental myocardial infarction. Circulation 2002;106:3126-3132.

5. Geng DF, Wu W, Jin DM, et al. Effect of peroxisome proliferatoractivated receptor gamma ligand. Rosiglitazone on left ventricular remodeling in rats with myocardial infarction. Int J Cardiol 2006;113: 86-91.

6. Zhao $\mathrm{C}$, Chen $\mathrm{W}$, Yang $\mathrm{L}$, et al. PPARgamma agonists prevent TGFbeta1/Smad3-signaling in human hepatic stellate cells. Biochem Biophys Res Commun 2006;350:385-391.

7. Marx N, Froehlich J, Siam L, et al. Antidiabetic PPAR gamma-activator rosiglitazone reduces MMP-9 serum levels in type 2 diabetic patients with coronary artery disease. Arterioscler Thromb Vasc Biol 2003;23:283-288.

8. Chen MM, Lam A, Abraham JA, et al. CTGF expression is induced by TGF- beta in cardiac fibroblasts and cardiac myocytes: a potential role in heart fibrosis. J Mol Cell Cardiol 2000;32:1805-1819.

9. Collins AR, Schnee J, Wang W, et al. Osteopontin modulates angiotensin II-induced fibrosis in the intact murine heart. J Am Coll Cardiol 2004;43:1698-1705.

10. Benson $\mathrm{SC}$, Pershadsingh $\mathrm{HA}, \mathrm{Ho} \mathrm{Cl}$, et al. Identification of telmisartan as a unique angiotensin II receptor antagonist with selective PPARgamma-modulating activity. Hypertension 2004;43:993-1002.

11. Schupp $M$, Janke J, Clasen $R$, et al. Angiotensin type 1 receptor blockers induce peroxisome proliferator-activated receptor-gamma activity. Circulation 2004;109:2054-2057.

12. Nagel JM, Tietz AB, Goke B, et al. The effect of telmisartan on glucose and lipid metabolism in nondiabetic, insulin-resistant subjects. Metabolism 2006:55:1149-1154.

13. Onai $Y$, Suzuki J, Maejima $Y$, et al. Inhibition of NF-\{kappa\}B improves left ventricular remodeling and cardiac dysfunction after myocardial infarction. Am J Physiol Heart Circ Physiol 2007;292:H530-H538. 
14. Onai Y, Suzuki J, Kakuta T, et al. Inhibition of IkappaB phosphorylation in cardiomyocytes attenuates myocardial ischemia/reperfusion injury. Cardiovasc Res 2004:63:51-59.

15. Matsuda T, Zhai P, Maejima Y, et al. Distinct roles of GSK-3alpha and GSK-3beta phosphorylation in the heart under pressure overload. Proc Natl Acad Sci USA 2008;105:20900-20905.

16. Maejima $\mathrm{Y}$, Adachi $\mathrm{S}$, Ito $\mathrm{H}$, et al. Induction of premature senescence in cardiomyocytes by doxorubicin as a novel mechanism of myocardial damage. Aging Cell 2008;7:125-136.

17. Maejima $\mathrm{Y}$, Adachi S, Morikawa K, et al. Nitric oxide inhibits myocardial apoptosis by preventing caspase-3 activity via S-nitrosylation. J Mol Cell Cardiol 2005;38:163-174.

18. Cowling RT, Gurantz D, Peng J, et al. Transcription factor NF-kappa B is necessary for up-regulation of type 1 angiotensin II receptor mRNA in rat cardiac fibroblasts treated with tumor necrosis factor-alpha or interleukin-1 beta. J Biol Chem 2002;277:5719-5724.

19. Mani K, Kitsis RN. Myocyte apoptosis: programming ventricular remodeling. J Am Coll Cardiol 2003:41:761-764.

20. Ashizawa N, Graf K, Do YS, et al. Osteopontin is produced by rat cardiac fibroblasts and mediates A(II)-induced DNA synthesis and collagen gel contraction. J Clin Invest 1996;98:2218-2227.

21. Hodsman GP, Kohzuki M, Howes LG, et al. Neurohumoral responses to chronic myocardial infarction in rats. Circulation 1988;78: 376-381.

22. Fraccarollo D, Galuppo P, Schmidt I, et al. Additive amelioration of left ventricular remodeling and molecular alterations by combined aldosterone and angiotensin receptor blockade after myocardial infarction. Cardiovasc Res 2005;67:97-105.

23. Milavetz JJ, Raya TE, Johnson CS, et al. Survival after myocardial infarction in rats: captopril versus losartan. J Am Coll Cardiol 1996;27:714-719.

24. Ju H, Zhao S, Jassal DS, et al. Effect of AT1 receptor blockade on cardiac collagen remodeling after myocardial infarction. Cardiovascul Res 1997;35:223-232.

25. Brown JD, Plutzky J. Peroxisome proliferator-activated receptors as transcriptional nodal points and therapeutic targets. Circulation 2007; 115:518-533.

26. Hirayama H, Sugano M, Abe N, et al. Troglitazone, an antidiabetic drug, improves left ventricular mass and diastolic function in normotensive diabetic patients. Int J Cardiol 2001;77:75-79.

27. Horio T, Suzuki M, Suzuki K, et al. Pioglitazone improves left ventricular diastolic function in patients with essential hypertension. Am J Hypertens 2005;18:949-957.

28. Dormandy JA, Charbonnel B, Eckland DJ, et al. Secondary prevention of macrovascular events in patients with type 2 diabetes in the PROactive Study (PROspective pioglitAzone Clinical Trial In macroVascular Events): a randomised controlled trial. Lancet 2005;366:1279-1289.
29. Imayama I, Ichiki $\mathrm{T}$, Inanaga $\mathrm{K}$, et al. Telmisartan downregulates angiotensin II type 1 receptor through activation of peroxisome proliferator-activated receptor gamma. Cardiovasc Res 2006:72:184-190.

30. Sabbah HN, Sharov VG, Lesch $M$, et al. Progression of heart failure: a role for interstitial fibrosis. Mol Cell Biochem 1995;147:29-34.

31. Morita $H$, Khanal S, Rastogi $S$, et al. Selective matrix metalloproteinase inhibition attenuates progression of left ventricular dysfunction and remodeling in dogs with chronic heart failure. Am J Physiol Heart Circ Physiol 2006;290:H2522-H2527.

32. Sharov VG, Sabbah HN, Ali AS, et al. Abnormalities of cardiocytes in regions bordering fibrous scars of dogs with heart failure. Int J Cardiol 1997;60:273-279.

33. Ducharme A, Frantz S, Aikawa M, et al. Targeted deletion of matrix metalloproteinase-9 attenuates left ventricular enlargement and collagen accumulation after experimental myocardial infarction. J Clin Invest 2000;106:55-62.

34. Hayashidani S, Tsutsui H, Ikeuchi M, et al. Targeted deletion of MMP-2 attenuates early LV rupture and late remodeling after experimental myocardial infarction. Am J Physiol Heart Circ Physiol 2003;285: H1229-H1235.

35. Peterson JT, Hallak $\mathrm{H}$, Johnson $\mathrm{L}$, et al. Matrix metalloproteinase inhibition attenuates left ventricular remodeling and dysfunction in a rat model of progressive heart failure. Circulation 2001;103:2303-2309.

36. Shinzato $T$, Ohya $Y$, Nakamoto $M$, et al. Beneficial effects of pioglitazone on left ventricular hypertrophy in genetically hypertensive rats. Hypertens Res 2007;30:863-873.

37. Oyama Y, Akuzawa N, Nagai R, et al. PPARgamma ligand inhibits osteopontin gene expression through interference with binding of nuclear factors to A/T-rich sequence in THP-1 cells. Circ Res 2002;90:348-355.

38. Nakamachi T, Nomiyama T, Gizard F, et al. PPARalpha agonists suppress osteopontin expression in macrophages and decrease plasma levels in patients with type 2 diabetes. Diabetes 2007;56: 1662-1670.

39. Yoshiji H, Kuriyama S, Yoshii J, et al. Angiotensin-Il type 1 receptor interaction is a major regulator for liver fibrosis development in rats. Hepatology 2001;34:745-750.

40. Arishiro K, Hoshiga M, Negoro N, et al. Angiotensin receptor-1 blocker inhibits atherosclerotic changes and endothelial disruption of the aortic valve in hypercholesterolemic rabbits. J Am Coll Cardiol 2007;49:1482-1489.

41. Li Y, Takemura G, Okada H, et al. Molecular signaling mediated by angiotensin II type $1 \mathrm{~A}$ receptor blockade leading to attenuation of renal dysfunction-associated heart failure. J Card Fail 2007;13:155-162.

42. Lods N, Ferrari P, Frey FJ, et al. Angiotensin-converting enzyme inhibition but not angiotensin II receptor blockade regulates matrix metalloproteinase activity in patients with glomerulonephritis. J Am Soc Nephrol 2003;14:2861-2872. 